\title{
Lipids and oxidative stress in blood serum of alloxan-induced diabetic rats: possible effects on liver and kidney tissues.
}

\author{
Benrebai $\mathbf{M}^{\mathrm{a}}$, Abidli $\mathbf{N}^{\mathrm{a}}$, Benlatreche $\mathbf{C}^{\mathrm{b}}$ \\ ${ }^{a}$ Département des sciences de la nature et de la vie. Université Mentouri, \\ Constantine 25000, Algeria. \\ ${ }^{\mathrm{b}}$ Laboratoire de biochimie, CHU Benbadis, Constantine 25000, Algeria.
}

\begin{abstract}
Experimental diabetes mellitus in rats was produced by a single dose of $200 \mathrm{mg}$ of alloxan $/ \mathrm{kg}$ body weight given intraperitoneally as an aqueous solution. The diabetic rats showed abnormal high blood glucose and variable increments in serum total lipids as well as in other serum lipid constituents particularly the triglycerides.

Certain pathological abnormalities could be detected in both liver and kidney, particularly when the disturbances in glucose and lipid metabolism were more pronounced in a group of diabetic rats that received no insulin treatment $\{$ NIT $\}$ or after four weeks of the onset of diabetes. On the other hand, putting the group of diabetic rats on an insulin regimen from the start of the diabetic condition resulted in a significant restoration of most evaluated parameters to values almost similar to those of normal control animals. Diabetic rats receiving no insulin treatment showed a markedly significant loss in body weight; and started to gain body weight gradually when put on the proper insulin regimen.
\end{abstract}

\section{Introduction}

Diabetes mellitus is a disease of different etiology, it may arise as a result of abnormalities in a number of intrinsic factors as stress; pregnancy or disorder in $\beta$ cells function in synthesis and secretion of insulin. Experimentally, the disease can be induced through the destruction of $\beta$ cells in the islets of Langerhans by diabetogenic agents such as alloxan. Much attention is given to studies on biochemical abnormalities that associate the disease as well as the proper control of its course, since if the diabetic condition is not well controlled a number of undesirable dangerous complications such as atherosc-lerosis, heart disease, retinopathy and hepatic histopathological changes could be frequently encountered among diabetic patents. The present study deals with investigations on biochemical abnormalities in blood glucose, serum total lipids and their main constituents namely: cholesterol, triglycerides, phospholipids together with follow up of pathological changes in both the liver and kidney.

Also, the value of introduction of insulin treatment at the onset of the diabetic condition, or at the later period after alleviating the diabetic state and on its complications was investigated.

\section{Material and methods}

Albino rats of both sexes three to five months of age and weighing 300 to $320 \mathrm{gm}$ were used. Animals were left to feed ad libitum. Rats were rendered diabetic through the administration of a single dose of alloxan (200 mg / Kg body weight) injected intrap-eritoneally. Blood glucose was estimated by the glucose oxidase method (Trinder, 1969), serum triglycerides, chole-sterol and phospholipids were determined by enzymatic colorimetric methods (Takayama 1977); (Wahlefeld 1974); (Flegg 1973) ;(Richmond 1973) while serum total lipids were estimated using sulfophosphovanilin method reported by Zoellner and Kirsch (1962).

Insulin when introduced was inję 25 ted subcutaneously using special insulin needles, and the doses adopted were 1.5 


\section{Benrebai M et al}

to $3 \mathrm{IU} /$ rat according to the extent of elevation in its blood glucose level.

Blood samples were collected at weekly intervals from the tail vein as described by Hofmann (1963) and serum was separated after blood clotting and analyzed at once. Fresh liver and kidney specimens were extracted from killed rats, fixed with Bouin Holland as described by Martoja and Martoja (1967) and stained with Haematoxilin and Eosin.

\section{Results}

Data for control non diabetic rats (ND rats) are given in Table (1). The data obtained for the different parameters (body weight, blood glucose, serum triglycerides, cholest-erol, phospholipids and total lipids) investigated in the different diabetic groups are given in Table (2).

In diabetic rats receiving no insulin treatment, there was an increase in all biochemical parameters with severe loss in body weight at the end of the $4^{\text {th }}$ week after the onset of the diabetic state (Table, 3). These abnormalities were accompanied with serious pathological alterations in their hepatocytes, marked by microsteatosis (Araya, et al. 2004), ectasis of lobular venous centers and cytoplasmic clarification. Also there were swelling of the epithelial cells of the proximal convoluted renal tubules (Moorhead, et al., 1986; Craven, et al., 1987) together with glomerular lesions (Mauer, et al., 1981; Mauer, et al., 1984; Ashim, et al., 1990). Besides the death rate among the diabetic rats left without insulin treatment was significantly high $\left(18.2 \%\right.$ by the end of the $12^{\text {th }}$ week from the onset of the disease, Table, 4).

Insulin administration particularly when given early at the onset of the diabetic state caused a significant drop in blood glucose and serum triglycerides to values approaching the normal levels. Also the levels of serum cholesterol and phospho-lipids showed pronounced decrements although remained slightly elevated above normal (Table, 5). Furthermore, all insulin treated diabetic rats gained body weight and showed net decreases in serum glucose and triglycerides while the other parameters fluctuated around the normal values (Table, 6). A marked decrease in death rate particu-larly among the diabetic group that received insulin treatment immediately after the onset of the diabetic condition was observed.

The activities and levels of enzymatic and non-enzymatic antioxidants of all animal groups are presented in Table 1. After induction of diabetes and before initiation of treatment all diabetic rats had a significant decrease in the activities and levels of all the studied parameters, with the exception of SOD being significantly elevated, as comp-ared with normal rats. Treatment with insulin for eight weeks resulted in a significant restoration in most of the evaluated parameters to values that were not different from those of normal control animals.

Hepatopathological changes were alleviated in diabetic rats receiving insulin treatment four weeks after the onset of diabetes and were completely prevented among the diabetic rats put on insulin regimen immediately after the disease.

However the pathological changes in the epithelial cells of proximal renal convoluted tubules were not alleviated under late, (after 4 weeks of the onset of diabetes), insulin treatment. Yet the renal abnormalities were not detected among the diabetic rats treated with insulin immediately after the onset of the diabetes (Lijun Sun et al., 2002). 
Table (1) : Levels of biochemical parameters in ND rats

\begin{tabular}{|c|c|c|c|c|c|c|}
\hline \multicolumn{7}{|c|}{ Biochemical parameters mg/dl } \\
\cline { 2 - 7 } & Weight(gm) & Glycemia & Triglycerides & Cholesterol & Phospholipids & Total Lipids \\
\hline Range & $141-321$ & $61-129$ & $34-106$ & $33-116$ & $81-192$ & $205-429$ \\
\hline Mean & 207 & 98 & 69 & 66 & 138 & 320 \\
\hline \pm SE & 7.2 & 3.1 & 2.5 & 2.5 & 1.8 & 4.1 \\
\hline
\end{tabular}

Mean \pm SD values of enzymatic and non-enzymatic antioxidants of control rats, and diabetic control and insulin-treated rats before and after 8 weeks of treatment with insulin.

\begin{tabular}{|c|c|c|c|c|c|}
\hline \multirow[t]{2}{*}{ Parametera } & \multirow[t]{2}{*}{ Control } & \multicolumn{2}{|c|}{ Diabetic control } & \multicolumn{2}{|c|}{ Insulin treated } \\
\hline & & Week 0 & Week 8 & Week 0 & Week 8 \\
\hline GPx (U/g Hb) & $12.5 \pm 2.50$ & $8.34 \pm 1.63^{\mathrm{b}}$ & $7.55 \pm 2.08^{\mathrm{a}}$ & $7.55 \pm 1.92^{\mathrm{a}}$ & $10.5 \pm 1.74^{\mathrm{c} \mathrm{d}}$ \\
\hline GRx (U/g Hb) & $4.40 \pm 0.27$ & $2.58 \pm 0.29^{\mathrm{a}}$ & $2.66 \pm 0.34^{\mathrm{a}}$ & $2.71 \pm 0.25^{\mathrm{a}}$ & $3.56 \pm 0.32^{\mathrm{b}}, \mathrm{c}$ \\
\hline SOD (U/g Hb) & $0.22 \pm 0.05$ & $0.46 \pm 0.04^{\mathrm{a}}$ & $0.44 \pm 0.07^{\mathrm{a}}$ & $0.44 \pm 0.08^{\mathrm{a}}$ & $0.25 \pm 0.04^{b}, c$ \\
\hline CAT (U/g Hb) & $1.12 \pm 0.30$ & $0.69 \pm 0.16^{\mathrm{a}}$ & $0.66 \pm 0.15^{\mathrm{a}}$ & $0.66 \pm 0.12^{\mathrm{a}}$ & $0.85 \pm 0.23^{b} c^{c}$ \\
\hline GSH (Amol/g Hb) & $4.55 \pm 0.50$ & $2.31 \pm 0.34^{\mathrm{a}}$ & $2.09 \pm 0.37^{\mathrm{a}}$ & $2.25 \pm 0.30^{\mathrm{a}}$ & $3.95 \pm 0.45^{\mathrm{b}}$, \\
\hline
\end{tabular}

a GPx:= glutathione peroxidase,

GRx:= glutathione reductase,

$\mathrm{SOD}=$ : uperoxide dismutase,

CAT: $:$ catalase,

$\mathrm{GSH}:=$ glutathione.

Table (2) :

\begin{tabular}{|c|c|c|c|c|c|c|}
\hline \multicolumn{7}{|c|}{ Levels of biochemical parameters in D rats 1st (week) } \\
\hline \multirow{5}{*}{$\begin{array}{c}\text { Range } \\
\text { Mean } \\
\pm \text { SE }\end{array}$} & \multirow[b]{2}{*}{ Weight(gm) } & \multicolumn{3}{|c|}{ Biochemical parameters $\mathrm{mg} / \mathrm{dl}$} & \multirow[b]{2}{*}{ Phospholipids } & \multirow[b]{2}{*}{ Total Lipids } \\
\hline & & Glycemia & Triglycerides & Cholesterol & & \\
\hline & $130-300$ & $246-466$ & $56-376$ & $24-102$ & $61-257$ & $308-704$ \\
\hline & 197 & 348 & 119 & 66 & 137 & 460 \\
\hline & 6.4 & 10.1 & 12.2 & 3.2 & 6.7 & 19.1 \\
\hline \multicolumn{7}{|c|}{ Levels of biochemical parameters in D rats 2nd (week) } \\
\hline \multirow{5}{*}{$\begin{array}{c}\text { Range } \\
\text { Mean } \\
\pm \text { SE } \\
\end{array}$} & & \multicolumn{3}{|c|}{ Biochemical parameters $\mathrm{mg} / \mathrm{dl}$} & & \multirow[b]{2}{*}{ Total Lipids } \\
\hline & Weight(gm) & Glycemia & Triglycerides & Cholesterol & Phospholipids & \\
\hline & $148-279$ & $460-.246$ & $66-210$ & $41-119$ & $80-255$ & $275-599$ \\
\hline & 191 & 320 & 125 & 73 & 140 & 415 \\
\hline & 5.4 & 9.1 & 7.4 & 3.8 & 7.6 & 21.7 \\
\hline \multicolumn{7}{|c|}{ Levels of biochemical parameters in D rats 3rd (week) } \\
\hline \multirow{5}{*}{$\begin{array}{l}\text { Range } \\
\text { Mean } \\
\pm \text { SE }\end{array}$} & & \multicolumn{3}{|c|}{ Biochemical parameters $\mathrm{mg} / \mathrm{dl}$} & & \multirow[b]{2}{*}{ Total Lipids } \\
\hline & Weight $(\mathrm{gm})$ & Glycemia & Triglycerides & Cholesterol & Phospholipids & \\
\hline & $143-259$ & $210-423$ & $72-252$ & $49-202$ & $93-207$ & $297-626$ \\
\hline & 184 & 325 & 137 & 76.2 & 134 & 411 \\
\hline & 5.4 & 11.5 & 7.6 & 5.3 & 5.1 & 14.7 \\
\hline \multicolumn{7}{|c|}{ Levels of biochemical parameters in D rats 4th (week) } \\
\hline \multirow{5}{*}{$\begin{array}{c}\text { Range } \\
\text { Mean } \\
\pm \mathrm{SE}\end{array}$} & & \multicolumn{3}{|c|}{ Biochemical parameters $\mathrm{mg} / \mathrm{dl}$} & & \multirow[b]{2}{*}{ Total Lipids } \\
\hline & Weight(gm) & Glycemia & Triglycerides & Cholesterol & Phospholipids & \\
\hline & $139-245$ & $211-424$ & $87-195$ & $46-88$ & $93-185$ & \multirow{3}{*}{$\begin{array}{c}333-528 \\
402 \\
9 \\
\end{array}$} \\
\hline & 178 & 310 & 135 & 68 & 136 & \\
\hline & 5.2 & 12.4 & 4.5 & 2.1 & 4.3 & \\
\hline
\end{tabular}

b p $<0.05$ as compared with control.

c $\mathrm{p}<0.05$ as compared with Week 16 (between groups).

$\mathrm{d} \mathrm{p}<0.01$ as compared with Week 0 (within group).

(1) 


\section{Benrebai M et al}

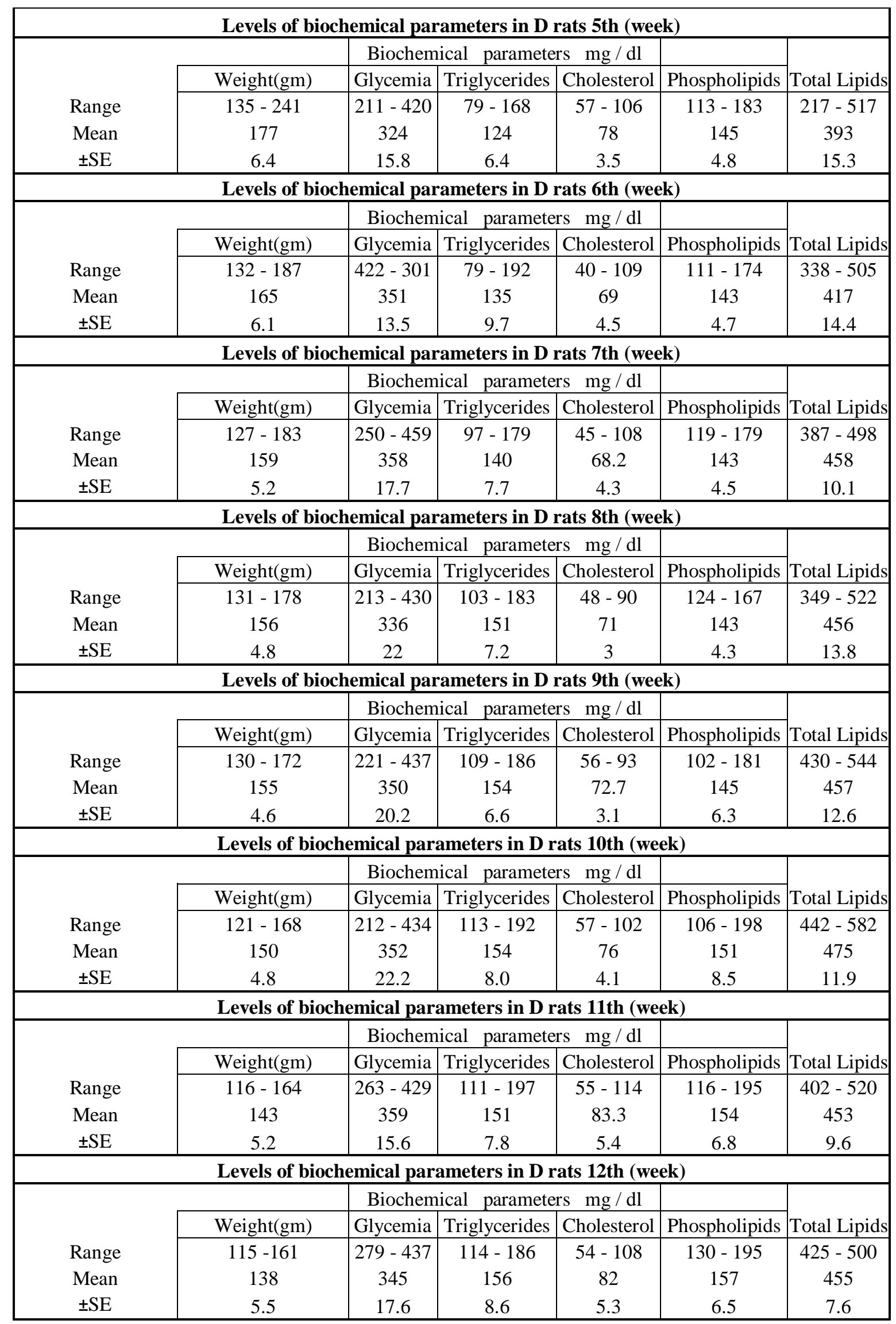


Table (3) : Statistical studies of biochemical parameters in D rats

\begin{tabular}{|c|c|c|c|c|}
\hline Weeks & 1 & 2 & 3 & 4 \\
\hline Nbr of rats & 33 & 30 & 28 & 27 \\
\hline \multicolumn{5}{|l|}{ Bio-parameters } \\
\hline $\begin{array}{l}\text { Weight range } \\
\text { Mean } \pm \mathrm{SE} \\
\text { Variation } \%\end{array}$ & $\begin{array}{c}130-300 \\
197 \quad 6 . . \\
4 \\
-40.0\end{array}$ & $\begin{array}{c}148-279 \\
1915.4 \\
-77.0\end{array}$ & $\mid$\begin{tabular}{cc}
\multicolumn{2}{c}{$143-259$} \\
184 & 5 \\
.4 & \\
& -11.1
\end{tabular} & $\begin{array}{c}139-245 \\
5.2 \\
-14.0\end{array}$ \\
\hline $\begin{array}{c}\text { Glycemia } \\
\text { Mean } \pm \mathrm{SE} \\
\text { Variation \% }\end{array}$ & $\begin{array}{c}246-466 \\
348 \quad 10.1 \\
+266\end{array}$ & $\begin{array}{c}460-.246 \\
320 \quad 9.1 \\
+227\end{array}$ & $\begin{array}{rl}210 & -423 \\
325 & 11.6 \\
& +232\end{array}$ & $\begin{array}{c}211-424 \\
31012.5 \\
+216\end{array}$ \\
\hline $\begin{array}{l}\text { Triglycerides } \\
\text { Mean } \pm \text { SE } \\
\text { Variation } \%\end{array}$ & $\begin{array}{c}56-376 \\
11912.2 \\
+72\end{array}$ & $\begin{array}{r}66-210 \\
1257 \\
5 \\
4 \\
126+ \\
8 \\
1\end{array}$ & $\begin{array}{c}72- \\
137 \\
7.6 \\
+99\end{array}$ & $\begin{array}{c}87-195 \\
135 \quad 4.5 \\
+96\end{array}$ \\
\hline $\begin{array}{l}\text { Cholesterol } \\
\text { Mean } \pm \text { SE } \\
\text { Variation \% }\end{array}$ & $\begin{array}{cc}24-102 \\
66 & 3 \\
& \\
& 2 \\
& \\
67 & \\
& + \\
& + \\
& 0 \\
& . \\
& 0\end{array}$ & 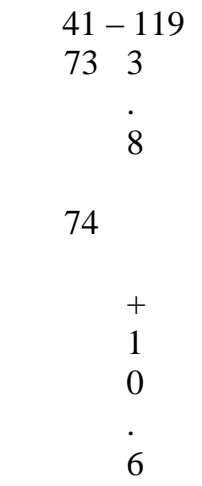 & $\begin{array}{cl}49-202 \\
76.2 \quad 5 . \\
& 3 \\
76.3 & + \\
& 15 \\
& .2\end{array}$ & $\begin{array}{c}46-88 \\
68 \quad 2 \\
\\
\quad \cdot \\
\\
+3.0\end{array}$ \\
\hline $\begin{array}{c}\text { Phospholipids } \\
\text { Mean } \pm \text { SE } \\
\text { Variation } \%\end{array}$ & $\begin{array}{c}61-257 \\
137 \quad 6.7 \\
-0.7\end{array}$ & $\begin{array}{cc}80-255 \\
140 \quad 7.6 \\
141+1.4\end{array}$ & 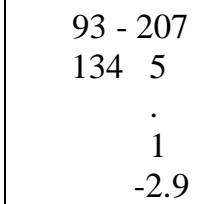 & $\begin{array}{c}93-185 \\
136 \quad 4.3 \\
-1.4\end{array}$ \\
\hline $\begin{array}{c}\text { Tot. Lipids } \\
\text { Mean } \pm \text { SE } \\
\text { Variation } \%\end{array}$ & $\begin{array}{c}308-704 \\
460 \quad 19.1 \\
+43.8\end{array}$ & $\begin{array}{cc}275 & -599 \\
415 & 2 \\
& 1 \\
& 5 \\
& 7 \\
416 & + \\
& 2 \\
9 & \\
& \\
& 7\end{array}$ & $\begin{array}{cc}297 & -626 \\
411 & 1 \\
& 4 \\
& \cdot \\
& 7 \\
412 & + \\
& 2 \\
& 8 \\
& . \\
& 4\end{array}$ & $\begin{array}{c}333-528 \\
4029.0 \\
+25.6\end{array}$ \\
\hline
\end{tabular}

$\mathrm{D}=$ diabetic rats

Table (4) : \% of death among D rats non treated with Insulin

\begin{tabular}{|c|c|c|c|c|c|c|c|c|c|c|c|c|}
\hline Weeks & 1 & 2 & 3 & 4 & 5 & 6 & 7 & 8 & 9 & 10 & 11 & 12 \\
\hline Remaining rats (week ) & 33 & 30 & 28 & $27 *$ & 20 & 15 & 12 & 12 & 12 & 12 & 11 & 9 \\
\hline Nbr of death (weekly) & 0 & 3 & 2 & 1 & 0 & 5 & 3 & 0 & 0 & 0 & 1 & 2 \\
\hline Rate of death / Past week \% & 0 & 9 & 6.7 & 3.6 & 0 & 25 & 20 & 0 & 0 & 0 & 8.3 & 18.2 \\
\hline
\end{tabular}

\footnotetext{
* 7 rats were withdrawn to be treated with insulin
} $\mathrm{D}=$ diabetic rats 


\begin{tabular}{|c|c|c|c|c|c|c|c|c|c|}
\hline Weeks & 0 & 1 & 2 & 3 & 4 & 5 & 6 & 7 & 8 \\
\hline Nbr of rats & 9 & 9 & 9 & 7 & 7 & 6 & 6 & 6 & 6 \\
\hline \multicolumn{10}{|l|}{ Bio-parameters } \\
\hline $\begin{array}{c}\text { Weight range } \\
\text { Mean } \pm \mathrm{SE} \\
\text { Variation } \% \\
\end{array}$ & \begin{tabular}{|ll}
143 & -204 \\
176 & 7.1 \\
\end{tabular} & \begin{tabular}{|c|}
$135-193$ \\
1666.7 \\
-5.7 \\
\end{tabular} & $\begin{array}{c}144-199 \\
1746.3 \\
-1.8 \\
\end{array}$ & $\begin{array}{c}155-200 \\
1796.4 \\
1.7 \\
\end{array}$ & $\begin{array}{c}161-216 \\
1877.2 \\
7.4 \\
\end{array}$ & $\begin{array}{c}168-227 \\
1928.9 \\
9.1 \\
\end{array}$ & $\begin{array}{c}171-232 \\
1989.4 \\
12.5\end{array}$ & \begin{tabular}{|c|}
$174-235$ \\
2039.7 \\
15.3
\end{tabular} & $\begin{array}{c}179-239 \\
2089.7 \\
10.2\end{array}$ \\
\hline $\begin{array}{c}\text { Glycemia } \\
\text { Mean } \pm \text { SE } \\
\text { Variation } \% \\
\end{array}$ & $\begin{array}{l}265-466 \\
355\end{array}$ & \begin{tabular}{|c|}
$52-105$ \\
785.9 \\
-75.0 \\
\end{tabular} & $\begin{array}{c}50-110 \\
806.8 \\
-77.9 \\
\end{array}$ & $\begin{array}{l}53-92 \\
705.3 \\
-80.5 \\
\end{array}$ & $\begin{array}{c}60-87 \\
683.8 \\
-80.8 \\
\end{array}$ & $\begin{array}{c}60-66 \\
630.8 \\
-82.3 \\
\end{array}$ & $\begin{array}{cc}59 & -69 \\
64 & 1.4 \\
-82.0 \\
\end{array}$ & $\begin{array}{c}60-79 \\
662.8 \\
-81.4 \\
\end{array}$ & $\begin{array}{c}55-75 \\
663.1 \\
-81.4 \\
\end{array}$ \\
\hline $\begin{array}{c}\text { Triglyceride } \\
\text { Mean } \pm \text { SE } \\
\text { Variation } \%\end{array}$ & $\begin{array}{ll}65 & -130 \\
81 & 6.6 \\
\end{array}$ & \begin{tabular}{|cc}
$46-101$ \\
$57 \quad 6.0$ \\
-29.6 \\
\end{tabular} & $\begin{array}{c}41-111 \\
587.0 \\
-28.4 \\
\end{array}$ & $\begin{array}{cc}40 & -65 \\
53 & 2.9 \\
-34.6 & \\
\end{array}$ & $\begin{array}{cc}45 & -67 \\
58 & 2.8 \\
-28.4 & \\
\end{array}$ & $\begin{array}{cc}49 & -71 \\
60 & 3.6 \\
-26.0 \\
\end{array}$ & $\begin{array}{cc}53 & -80 \\
63 & 3.6 \\
-22.3 \\
\end{array}$ & $\begin{array}{cc}58 & -83 \\
68 & 3.3 \\
-16.0 \\
\end{array}$ & $\begin{array}{c}62-80 \\
68 \quad 2.8 \\
-16.0 \\
\end{array}$ \\
\hline $\begin{array}{c}\text { Cholesterol } \\
\text { Mean } \pm \text { SE } \\
\text { Variation \% }\end{array}$ & \begin{tabular}{|ll}
70 & -102 \\
90 & 3.8
\end{tabular} & \begin{tabular}{|c|}
$68-108$ \\
904.8 \\
0.0
\end{tabular} & $\begin{array}{c}64-103 \\
874.5 \\
-3.3 \\
\end{array}$ & $\begin{array}{ll}60 & -88 \\
71 & 3.2 \\
-21.1 & \\
\end{array}$ & $\begin{array}{c}64-82 \\
732.2 \\
-18.9 \\
\end{array}$ & $\begin{array}{l}62-80 \\
722.6 \\
-20.0\end{array}$ & $\begin{array}{cc}68-90 \\
772.9 \\
-14.5 \\
\end{array}$ & $\begin{array}{c}70-90 \\
792.6 \\
-12.2 \\
\end{array}$ & $\begin{array}{c}72-94 \\
793.1 \\
-12.2 \\
\end{array}$ \\
\hline $\begin{array}{c}\text { Phospholipids } \\
\text { Mean } \pm \text { SE } \\
\text { Variation \% }\end{array}$ & $\begin{array}{l}125-170 \\
153\end{array}$ & $\begin{array}{c}130-181 \\
1605.8 \\
4.5\end{array}$ & $\begin{array}{c}145-196 \\
1716.1 \\
11.8\end{array}$ & $\begin{array}{c}113-168 \\
1507.6 \\
-2.0 \\
\end{array}$ & $\begin{array}{c}117-198 \\
1529.3 \\
-0.7\end{array}$ & $\begin{array}{c}116-158 \\
1336.2 \\
-13.1 \\
\end{array}$ & \begin{tabular}{|c|}
$118-140$ \\
1293.4 \\
-15.7
\end{tabular} & \begin{tabular}{|c|}
$129-140$ \\
132 \\
13.2 \\
13.2
\end{tabular} & $\begin{array}{c}121-140 \\
1312.6 \\
-14.4\end{array}$ \\
\hline $\begin{array}{l}\text { Tot. Lipids } \\
\text { Mean } \pm \text { SE } \\
\text { Variation \% }\end{array}$ & $\begin{array}{rrr}390 & -485 \\
438 & 11.8\end{array}$ & $\mid \begin{array}{c}306-429 \\
37214.7 \\
-15.1\end{array}$ & 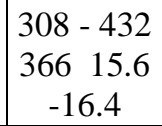 & $\begin{array}{c}327-437 \\
375 \quad 17.1 \\
-14.4\end{array}$ & $\begin{array}{c}339-430 \\
38217.7 \\
-12.8\end{array}$ & $\begin{array}{c}340-471 \\
37320.0 \\
-14.8\end{array}$ & $\begin{array}{cc}350 & -452 \\
381 & 15.0 \\
-13.1\end{array}$ & $\left|\begin{array}{cc}360 & -470 \\
390 & 17.0 \\
-11.0\end{array}\right|$ & $\begin{array}{c}365-412 \\
3807.5 \\
-13.2\end{array}$ \\
\hline
\end{tabular}

Table (5) : Insulin treated group after 4 weeks of the onset of the disease

Table (6) :Insulin treated group immediately after the onset of the disease 


\begin{tabular}{|c|c|c|c|c|c|c|c|c|c|}
\hline Weeks & 0 & 1 & 2 & 3 & 4 & 5 & 6 & 7 & 8 \\
\hline Nbr of rats & 7 & 7 & 5 & 5 & 4 & 4 & 3 & 3 & 3 \\
\hline \multicolumn{10}{|l|}{ Bio-parameters } \\
\hline $\begin{array}{c}\text { Weight range } \\
\text { Mean } \pm \text { SE } \\
\text { Variation } \%\end{array}$ & $\begin{array}{cr}153 & -191 \\
176 & 5.4\end{array}$ & $\begin{array}{c}162-199 \\
1855.1 \\
5.1\end{array}$ & $\begin{array}{c}167-198 \\
1855.9 \\
5.1\end{array}$ & $\begin{array}{c}172-203 \\
1906.1 \\
7.9\end{array}$ & \begin{tabular}{|c|}
$176-208$ \\
1938.0 \\
9.6
\end{tabular} & $\begin{array}{|cc|}182-213 \\
1987.8 \\
12.5\end{array}$ & $\begin{array}{c}186-217 \\
20513.9 \\
16.4\end{array}$ & $\begin{array}{c}191-220 \\
219 \mathrm{~ns} \\
24.4\end{array}$ & $\begin{array}{c}197-225 \\
214 \mathrm{~ns} \\
21.6\end{array}$ \\
\hline $\begin{array}{c}\text { Glycemia } \\
\text { Mean } \pm \text { SE } \\
\text { Variation \% }\end{array}$ & $\begin{array}{cc}235 & -353 \\
289 & 4.5\end{array}$ & $\begin{array}{c}61-137 \\
8110.5 \\
71.9 \\
\end{array}$ & $\begin{array}{ccc}52 & -104 \\
78 & 10.9 \\
74.0\end{array}$ & $\begin{array}{c}64-124 \\
9112.7 \\
68.5 \\
\end{array}$ & $\begin{array}{c}63-83 \\
724.3 \\
75.0 \\
\end{array}$ & $\begin{array}{c}65-81 \\
743.9 \\
74.3 \\
\end{array}$ & \begin{tabular}{|c|}
$52-104$ \\
$84 \mathrm{~ns}$ \\
70.9 \\
\end{tabular} & \begin{tabular}{|c|}
$60-102$ \\
$80 \mathrm{~ns}$ \\
72.3 \\
\end{tabular} & $\begin{array}{c}65-80 \\
71 \mathrm{~ns} \\
75.4 \\
\end{array}$ \\
\hline $\begin{array}{c}\text { Triglyceride } \\
\text { Mean } \pm \text { SE } \\
\text { Variation \% }\end{array}$ & $\begin{array}{c}107-175 \\
141 \quad 12\end{array}$ & $\begin{array}{c}48-86 \\
675.0 \\
52.4\end{array}$ & $\begin{array}{cc}53-74 \\
66 \quad 3.5 \\
53.2\end{array}$ & $\begin{array}{c}44-68 \\
58 \quad 4.4 \\
58.8\end{array}$ & $\begin{array}{c}54-74 \\
614.4 \\
56.7\end{array}$ & $\begin{array}{c}40-63 \\
592.0 \\
58.1\end{array}$ & \begin{tabular}{|c|}
$62-69$ \\
643.39 \\
54.6 \\
\end{tabular} & $\begin{array}{c}46-62 \\
56 \mathrm{~ns} \\
60.2\end{array}$ & $\begin{array}{c}45-65 \\
57 \mathrm{~ns} \\
59.6\end{array}$ \\
\hline $\begin{array}{l}\text { Cholesterol } \\
\text { Mean } \pm \text { SE } \\
\text { Variation } \%\end{array}$ & $\begin{array}{ll}46 & -80 \\
66 & 4.7\end{array}$ & $\begin{array}{cc}67 & -89 \\
80 & 3.1 \\
21.9\end{array}$ & $\begin{array}{c}59-81 \\
693.8 \\
4.5 \\
\end{array}$ & $\begin{array}{c}50-77 \\
634.4 \\
-4.5 \\
\end{array}$ & $\begin{array}{c}51-88 \\
677.9 \\
1.5 \\
\end{array}$ & $\begin{array}{c}50-74 \\
635.4 \\
-4.5 \\
\end{array}$ & $\begin{array}{|cc|}49 & -73 \\
57 & 10.8 \\
-13.6 \\
\end{array}$ & $\begin{array}{c}66-80 \\
72 \mathrm{~ns} \\
9.0 \\
\end{array}$ & $\begin{array}{c}66-105 \\
81 \mathrm{~ns} \\
22.7 \\
\end{array}$ \\
\hline $\begin{array}{c}\text { Phospholipids } \\
\text { Mean } \pm \text { SE } \\
\text { Variation } \%\end{array}$ & $\begin{array}{cc}93 & -156 \\
156 & 7.6\end{array}$ & $\begin{array}{c}124-173 \\
1546.8 \\
22.2\end{array}$ & $\begin{array}{c}109-166 \\
13010.0 \\
3.1\end{array}$ & $\begin{array}{c}63-184 \\
12521.0 \\
-0.8\end{array}$ & \begin{tabular}{|c|}
$118-150$ \\
1327.4 \\
4.7
\end{tabular} & \begin{tabular}{|c|}
$98-130$ \\
1187.5 \\
-6.3 \\
\end{tabular} & $\begin{array}{c}100-113 \\
1055.7 \\
-16.6\end{array}$ & \begin{tabular}{|c|}
$112-128$ \\
$121 \mathrm{~ns}$ \\
-3.9 \\
\end{tabular} & $\begin{array}{c}130-150 \\
138 \mathrm{~ns} \\
9.5\end{array}$ \\
\hline $\begin{array}{l}\text { Tot. Lipids } \\
\text { Mean } \pm \text { SE } \\
\text { Variation } \% \\
\end{array}$ & $\begin{array}{rr}333 & -428 \\
382 & 12.4\end{array}$ & $\begin{array}{c}337-408 \\
3748.7 \\
2.1 \\
\end{array}$ & $\begin{array}{c}317-441 \\
34428.3 \\
9.9 \\
\end{array}$ & \begin{tabular}{|c|}
$238-464$ \\
35236.2 \\
7.8 \\
\end{tabular} & \begin{tabular}{|c|}
$232-429$ \\
33143.2 \\
13.3 \\
\end{tabular} & \begin{tabular}{|c|}
$295-380$ \\
34420.6 \\
9.9 \\
\end{tabular} & \begin{tabular}{|c|}
$266-421$ \\
3647.0 \\
4.7 \\
\end{tabular} & \begin{tabular}{|c|}
$285-385$ \\
$337 \mathrm{~ns}$ \\
11.7 \\
\end{tabular} & $\begin{array}{c}297-403 \\
356 \mathrm{~ns} \\
6.8 \\
\end{array}$ \\
\hline
\end{tabular}

\section{Discussion}

Intraperitoneal administration of single dose of alloxan (200 mg / Kg BW) led to the development of a severe diabetic state (Marliss et al., 1981) 48 to 72 hours after alloxan administration accompanied by a significant loss in body weight. The latter could be attributed to disturbances in metabolism associated in insulin deficiency (Davidson and Kaplan, 1977).

Also, it was accompanied with a high percent of death, which had been attributed principally to hypoglycemic phase after alloxan administration as noted and suggested by (Okamoto, 1970)

High death continued during the $1^{\text {st }}$ $2^{\text {nd }}$ and $3^{\text {rd }}$ weeks after the onset of diabetes (Garcia, et al 1974). but such rat was significantly lower when insulin treatment was initiated at the onset of the disease (Table 4).

The diabetic hyperglycemia due to insulin deficiency was accompanied with hyper triglyceridemia, an observation also reported by Bragdon and Gordon (1958), and Grundy et al. (1979). The increase in serum triglycerides seems to be due to a number of intrinsic factors such as a decrease of triglyceride uptake by adipose tissue, the intensified triglycerides 251 production and release by the liver (Nestel and Steinberg, 1963; Van Tol, 1977; Weiland, et al., 1980).

Elevation in serum cholesterol in the alloxan diabetic rats appeared not be slightly related to the extent of hyperglycemia, most probably to metabolic disturbances corre-lated with abnormalities in the function of enzymatic systems concerned with synthesis and breakdown of cholesterol mainly HMG reductase and HMG synthase (Anderson, et al., 1994; Thomson, 1980).

Besides the accelerated absorption of cholesterol by the intestine has been suggested (Zhang, and Beynen, 1993) to play an important role in the causation of this hypercholesterolemia among diabetic rats. The minor changes in serum phospholipids seems to be related to the disturbances in the different phospholipase enzymatic activities (Kunz, et al., 1994); under the effect of insulin deficiency as well as the concomitant abnormalities in glucose metabolism.

Enforcement of insulin treatment, four weeks after the development of diabetic state, decreased to a limited exte$\mathrm{nt}$, the rate of death, while when insulin 


\section{Benrebai M et al}

treatment was adopted immediately after the onset of the disease the rate of death was significantly decreased concomitant with disappearance of pronounced biochemical abnormalities in serum and pathological abnormalities in liver and kidney (Wexler, 1970; Stout, 1979; Wright et al., 1980; Wright et al., 1983; Hostetter, 1985; Holthofer, et al., 1987).

In this study it is suggested that the biochemical changes in glucose and lipid metabolism that accompany diabetes resulting from insulin deficiency could participate in the development of different undesirable complications affecting various organs, among which are the liver and kidney and endangering the life of patients. Besides, it reveals that insulin treatment and well control of the diabetic conditions, as early as possible from the onset of the disease, could significantly lower the risk of the development of such complications.

\section{References}

1. Anderson J W, Jones A E and Riddell-Mason S (1994): Ten different dietary fibers have significantly different effects on serum and liver lipids of cholesterol fed rats. J. Nutr. 124: 78.

2. Araya $\mathbf{J}$, Rodrigo R, Videla $\mathbf{L}$, Thielemann L, Orellana M, Pettinelli $\mathbf{P} \&$ Poniachik J (2004): Increase in long-chain polyunsaturated fatty acid $n-6 / n$ 3 ratio in relation to hepatic steatosis in non-alcoholic fatty liver disease patients. (Jan 13, 2004) Clin. Sci. (Lond.) 10.1042/ CS20030326 [Epub ahead of print].

3. Ashim K Sinha, et al (1990) Effects of fish oil on glomerular function in rats with diabetes mellitus. J Lipid Res.. 31: 1219.

4. Bragdon J Hand Gordon RS (1958)-Tissue distribution of $\mathrm{C}^{*}$ after the intrav-enous injection of labeled chylomicrons and unesterified fatty acids in the rat. J Clin. Invest. 37: 574

5. Craven $\mathbf{P}$ A, Caines $M A$ and De Rubertis F $R$ (1987). Sequential alterations in glomerular prostaglandin and thromboxane synthesis in diabetic rats: relationship to the hyperfiltration of early diabetes. Metabolism. 36: 95.

6. Davidson H B and Kaplan S A. (1977)-Increased insulin binding by hepatic plasma membranes from diabetic rats. Normalisation by insulin therapy. The journal of clinical investigation.59, 22.

7. Flegg H M (1973)Determination of serum cholesterol by an enzymic method. Ann. Clin. Biochem. 10 (3), 79.

8. Garcia M J P M, McNamara T, Gordon and W B Kannel (1974). Morbidity and mortality in diabetics in the Framingham population. Diabetes. 23: 105.

9. Grundy SM, Mok HYI, Zech LA, et al. (1979) Transport of very low density lipoproteintriglycerides in varying degrees of obesity and hypertriglyceridemia. J Clin Invest;63: 1274.

10. Hofmann G (1960)- Les animaux de laboratoire. (Précis) Vigot-frères Paris.

11. Holthofer H E, Pettersson and T Tornroth (1987). Diabetes mellitus-associated changes in glomerular glycocompounds. Histochem. J. 19: 351.

12. Hostetter T H (1985). Diabetic nephropathy. N. Engl. J. Med. 312: 642.

13. Kunz F, Pechlener C, Erhart $R$ and Muhberger $V$ (1994) HDL and plasma phospholipids in coronary artery disease. Atheroscler. Thromb. 14: 1146.

14. Lijun Sun $\neq$, et al (2002) Role of Sterol Regulatory Elementbinding Protein 1 in Regulation of Renal Lipid Metabolism and Glomerulosclerosis in Diabetes

15. Mellitus* The Journal of Biological Chemistry. Vol. 277, No. 21, Issue of May 24, pp. 18919.

16. Marliss, E. B., A. A. F. Sima, and A. F. Nakhooda. (1981). Spontaneous diabetes in the 'BB' Wistar rat. In Etiology and Pathogenesis of Insulin dependent Diabetes Mellitus.J. M. Martin, R. M. Ehrlich, and F. 
J. Holland, editors. Raven Press, New York. 251-.

17. Martoja, R. and Martoja P. M. - (1967) Initiation aux techniques d'histologie animale. Edt. Masson et Cie.

18. Mauer, S. M., M. W. Steffes, and D. M. Brown. (1981). The kidney in diabetes. Am. J. Med. 70: 603 .

19. Mauer, S. M., M. W. Steffes, E. N. Ellis, D. E. Sutherland, D. M. Brown, and F. C. Goetz. (1984). Structural-functional relationships in diabetic nephropathy. J. Clin. Invest. 74: 1143.

20. Moorhead, J. F., M. K. Chan, and $Z$. Varghese. 1986. The role of abnormalities of lipid metabolism in the progression of renal disease. Conhap. Issues Nephrol. 14: 133.

21. Nestle , P. J. and Steinberg, D. (1963) -Fate of palmitate and of linoleate perfused through the isolated rat liver at high concentration. J. Lip. Research., 4: 461 .

22. Okamoto, K. (1970) Experimental production of diabetes. Diabetes mellitus, Theory and practice. Ellemberg, $M$ and Rifkin, H. Diabetes mellitus arold theory and practice. Mac graw Hill. New York.230.

23. Richmond, W. (1973), Preparation and properties of a cholesterol oxidase from Nicardia species, and its application to the enzymic assay of total cholesterol in serum. Clin. Chem.19 (12) 1350.

24. Stout, R. W. (1979). Diabetes and atherosclerosis-the role of insulin. Diabetologiu. 16141.

25. Takayama , M.; et al - (1977),A new enzymic method for determination of serum choline containing phospholipids. Clin. Chim. Acta.7, (1) 93.

26. Thibert. (1983). Pathological lesions in the spontaneously diabetic BB Wistar rat: a comprehensive autopsy study. Metabolism. Sf (Supp1. 1) 101.

27. Thomson, A. B. R. (1980) Unidirectional flux rate of cholesterol and fatty acids into the intestine of rats with druginduced diabetes mellitus: effect of variations in the effective resistance of the unstirred water layer and the bile acid micelle. Journal of Lipid Research Volume 21, 687.

28. Trinder, P.(1969)

Determination of blood glucose using an oxidase Peroxidase system a non carcinogenic chromogens. Ann. Clin. Biochem. 6: 24.

29. Van Tol, A. (1977). Hypertriglycerid-emia in the diabetic rat. Defective removal of serum very low density lipoproteins. Atherosclerosis. 26 117.

30. Wahlfeld, A W (1974) - Dans H.U. Bergmeyer, Methoden der enzymatischen annalyse, $3^{\text {rd }}$ édition Tome II Velarg. Chemic Weinheim, p.

31. Weiland, D, C. E. Mondon, and G. M. Reaven. (1980). Evidence for multiple causality in the development of diabetic hypertriglyceridaemia. Diabetologia.18: 335.

32. Wexler, B. C. (1970). Histopathologic responses to severe alloxan diabetes in atherosclerotic and nonatherosclerotic rats. Diabetes. 19: 324.

33. Wright, J. R., Jr., A. J. Yates, N. T. Shah, J. C. Neff, D. W. Covey, and P. Thibert. (1980). Histological charact-eristics of the BB Wistar rat. Vet. Clin. Pathol. 14: 9.

34. Wright, J. R., Jr., A. J. Yates, H. M. Sharma, and P. Thibert. (1983). Pathological lesions in the spontaneously diabetic BB Wistar rat: a comprehensive autopsy study. Metabolism. Sf (Supp1. 1): 101.

35. Zhang, X. \& Beynen, A. C. (1993) Influence of dietary fish proteins on plasma and liver cholesterol concent-rations in rats. Br. J. Nutr. 69: 767.

36. Zoellner $\mathbf{N}$. and Kirsch, $\mathbf{K}$. (1962) - Microdetermination of lipids by the sulfophosphovanilin reaction. Ges. Exp. Med. 135, (6) 545. 


\section{Benrebai M et al}

الإجهاد التأكسدي في مصل دم الفئران المصابة بمرض السكر التجريبي بالألوكسان: إمكانية التأثير على نسيجي الكبان و الكليتين .

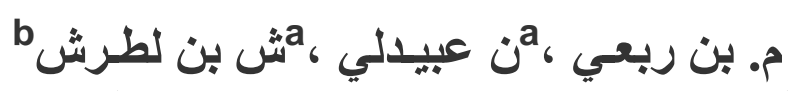

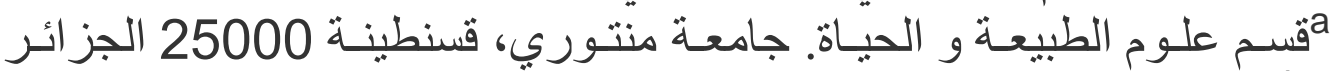
bعمل الكيمياء الحيوية المستشفى المركزي الجامعي إين باديس قسنطينة 25000 الجزائر الجائر

بعد ظهور ارتفاع ملحوظ في مستوى جلوكوز الدم عند الفئران المحقونة

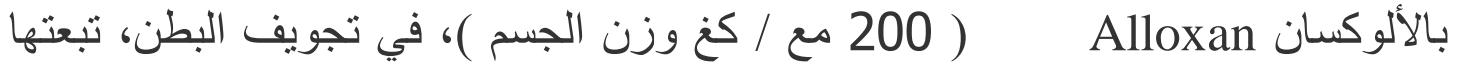

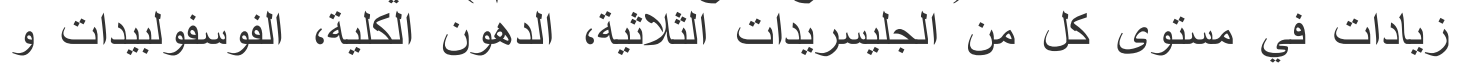

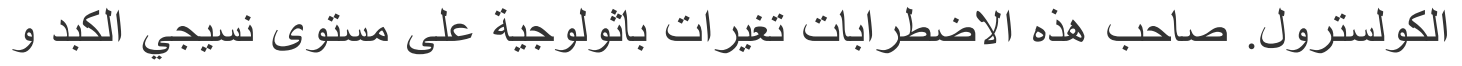

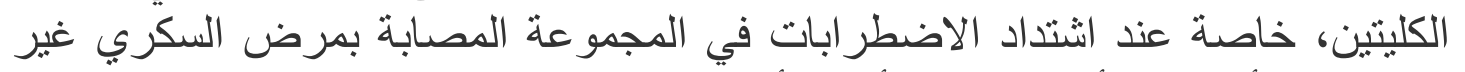
المعالجة بالأنسولين، أو بعد مضي أربعة أسابيع من حدوث المرضي المرض.

من ناحية أخرى المجموعة الدصابة بالمرض الخاضعة للعلاج بالأنسولين فور

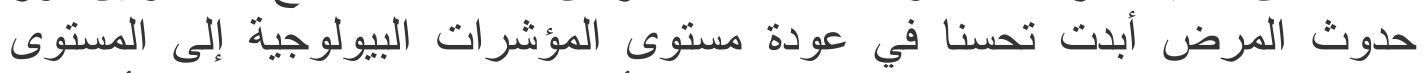

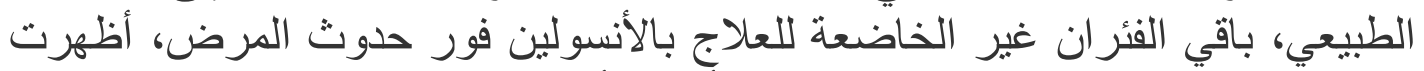

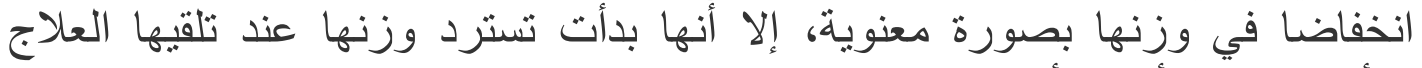

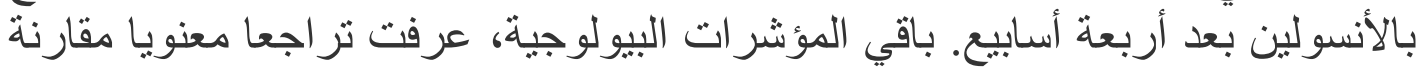

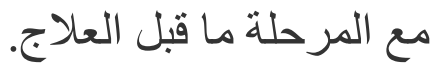

